\title{
DIGITAL TECHNOLOGY Rapid deployment of virtual ICU support during the COVID-19 pandemic
}

\author{
Authors: Adam Igra, ${ }^{\mathrm{A}}$ Helen McGuire, ${ }^{\mathrm{B}}$ Ian Naldrett, ${ }^{\mathrm{C}}$ Rosie Cervera-Jackson, ${ }^{\mathrm{D}}$ Rebecca Lewis, ${ }^{\mathrm{E}}$ Cliff Morgan ${ }^{\mathrm{F}}$ and \\ Louit Thakuria $^{\mathrm{G}}$
}

\begin{abstract}
The COVID-19 pandemic brought many serious challenges to the clinical workplace, and was a catalyst to novel approaches to the way in which we practice medicine. These challenges include extreme numbers of critically ill patients overwhelming many intensive care units, how to maintain the flow of communication between clinicians, patients and their families, and how to prevent the spread of infection working on quarantined units in personal protective equipment.

The Royal Brompton and Harefield Hospitals deployed a series of digital solutions to try to address some of those challenges and a series of case studies describes their clinical application in three clinical domains: communicating with families, clinical communication between clinicians and the delivery of clinical education.
\end{abstract}

KEYWORDS: COVID-19, intensive care, digital transformation

DOI: $10.7861 /$ fhj.2020-0157

\section{Introduction}

The Royal Brompton \& Harefield NHS Foundation Trust combines two west-London hospitals providing tertiary cardiothoracic care, including critical care with extracorporeal membrane oxygenation (ECMO) support. As such, the trust forms an integral part of the regional, and indeed national, healthcare system's response to the COVID-19 pandemic. Requirements from NHS regulators, led the trust to significantly increase its level 3 critical care capacity for the mechanical ventilation of COVID-19-positive patients. The trust has cared for 207 COVID-19 patients at the time of

Authors: Atransformation director, Royal Brompton \& Harefield

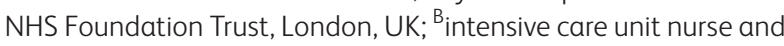
assistant nursing information officer, Royal Brompton \& Harefield NHS Foundation Trust, London, UK; Clead nurse for intensive care education, Royal Brompton \& Harefield NHS Foundation Trust, London, UK; D practice educator (adult intensive care), Royal Brompton \& Harefield NHS Foundation Trust, London, UK;

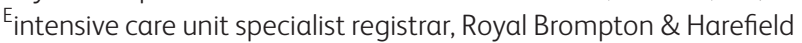
NHS Foundation Trust, London, UK; ${ }^{F}$ consultant in critical care and chief clinical information officer, Royal Brompton \& Harefield NHS Foundation Trust, London, UK; ${ }^{G}$ consultant in critical care, Royal Brompton \& Harefield NHS Foundation Trust, London, UK writing, and most of those patients were critically ill. Added to the clinical demand placed on the organisation were the challenges of personal protective equipment (PPE), availability and limitations on staff movement, difficulties in communication, and prohibition of visitors to minimise infection risks. This extraordinary situation necessitated radically different ways of working; we present some of the challenges we faced, with case examples to demonstrate the rapid deployment of a video-enabled digital communication infrastructure.

\section{Challenges of COVID-19 on critical care}

During the height of the surge in COVID-19 activity in April 2020, the Royal Brompton Hospital site was caring for four times as many critically ill patients (70) as its usual capacity across the intensive care unit (ICU) capacity (18 beds). Of these, 27 patients were supported with veno-venous ECMO at the peak of the surge, compared with the regular commissioned capacity of four ECMO beds in the unit. Similarly, Harefield Hospital accommodated 45 COVID-19-positive critical care patients at its peak, while serving as one of only two London centres for cardiac surgery that continued to operate through the pandemic.

In March, the surge in critically unwell patients demanded a rapid review of bed capacity, support to staff and optimising lines of communication. The ICU bed base was expanded by displacing designated paediatric intensive care, high-dependency and postoperative ward-level care spaces. This extension of adult intensive care to create surge areas warranted reassurance that their existing clinical teams would have readily available access to adult intensive care specialists for clinical advice. The expansion required rapid changes in our workforce, with doctors and nurses redeployed to critical care from all other specialties in the hospital. ${ }^{1}$ While we were able to provide additional preparatory critical care training, we also needed to provide those redeployed frontline workers with the appropriate clinical supervision from an increasingly stretched ICU team. This required clear communication channels within and across the ward teams. Firstly, to enable clinicians within the 'red-zones' to speak to colleagues in 'green-zones' for guidance and, secondly, to endorse amendments to clinical care and ongoing organ support. To facilitate this interaction and guide colleagues delivering essential critical care, senior intensivists required access to key information, including ventilator wave forms, that are not visible remotely on electronic patient records. Traditional telephones proved impractical for clinicians in red zones donned in PPE necessitating a hands-free communication solution in a ward environment. 
The delivery of compassionate as well as clinically excellent care is also one of our highest priorities. While hospital visits were curtailed as part of our infection control measures, there was an urgent need to help relatives to see their critically ill loved ones receiving care on our units, especially for those patients who were not expected to survive. Our clinical staff also wanted families to see that their loved ones were comfortable and well cared for.

Together, these challenges of the COVID-19 pandemic supported the need for a rapidly implemented and easily adoptable videoenabled digital communication infrastructure. Although the concept of telemedicine in critical care and 'virtual intensive care units' is not a new one, it had not been previously deployed at the Royal Brompton \& Harefield NHS Foundation Trust. 'Schwamm et al described the use of an in-room video intercom system to minimise PPE consumption and reduce the risks of nosocomial COVID-19 infection, and the rapid deployment of telemedicine solutions in response to COVID-19 have also described by other groups in the NewYork-Presbyterian and Stanford Health Care systems. ${ }^{2-4}$

\section{Innovating at pace}

Ordinarily the installation of a video-enabled digital communication infrastructure would take considerable time and investment. A practical solution was proposed that could be deployed in a matter of days at a time in response to clinical need during emergency circumstances. Microphone-enabled USB HD webcams were installed at every bedside computer. This used commercially available and General Data Protection Regulation (GDPR) compliant teleconferencing software (MS Teams (Microsoft, Redmond, USA)) to rapidly establish virtual ICU support for frontline workers (Fig 1a). Once installed, other applications for the platform have also been borne out including bedside participation in multidisciplinary team meetings and nurse practice education, as outlined in the following case examples.

\section{Open channels of communication at the bedside}

Using the video conferencing platform, a specific identity for each individual bed-space computer terminal was created alongside generic identities for each ward's designated 'red-zone' and 'green-zone' doctor and nurse. This allowed the platform to run continuously, creating an 'open-channel' of direct real-time and hands-free communication with each bed space in quarantined red-zones (Fig 1b), while the webcams offered live video-feeds of ventilator waveforms and patient monitors as required (Fig 1c). This simple low-cost intervention allowed a digital network between frontline clinical staff to be swiftly established.

\section{Case examples}

We present a series of case examples and testimonials that describe the use of this system to support clinical work through the challenges of the COVID-19 pandemic across three key areas. Case example 1 describes video calls with family members unable to visit quarantined patients due to infection control risks and travel restrictions, a situation especially heart-breaking at the end of life. Case example 2 describes how senior clinicians have provided specialist guidance and support to colleagues caring for complex patients from outside of quarantined red zones. Case example 3 describes how the system has been used to deliver critical care education for clinical staff redeployed to the ICU.
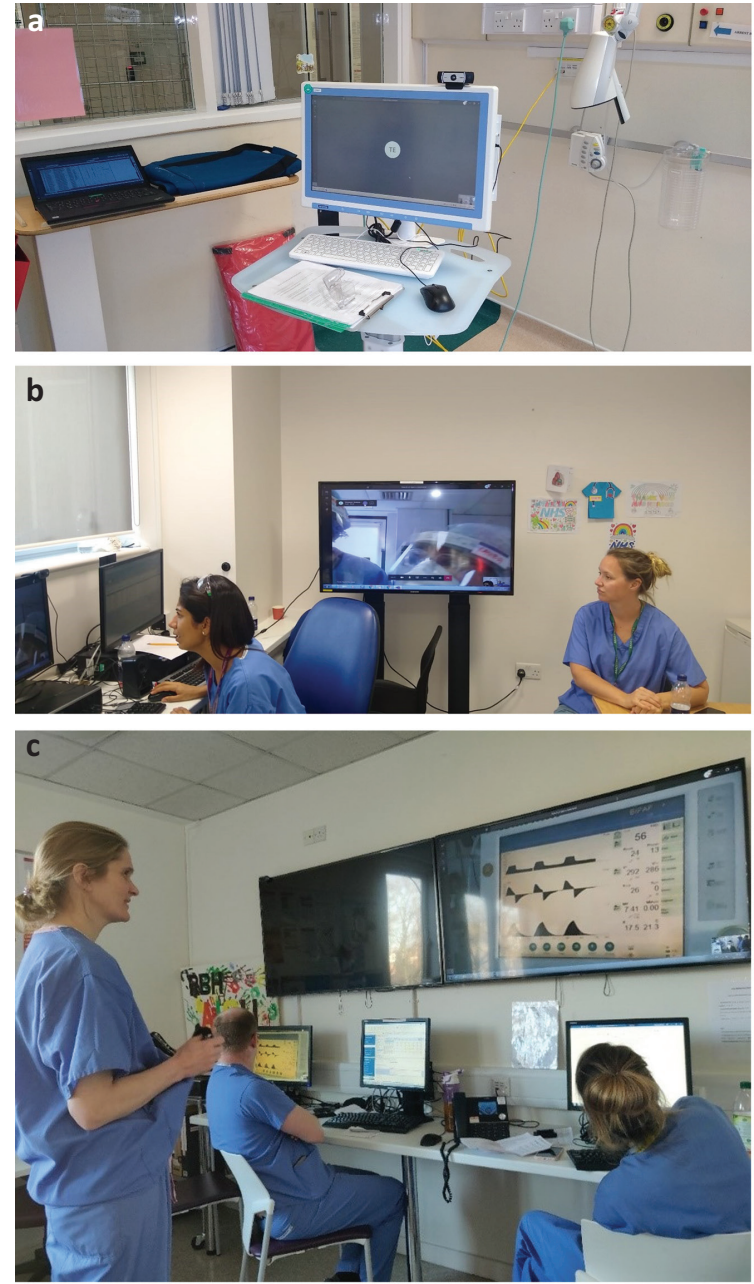

Fig 1. a) Webcam and conferencing software-enabled workstation to establish virtual ICU support for frontline workers. b) Webcam-enabled workstation providing live hands-free video communication with staff members working in quarantined red-zones. c) Webcam offering real-time video-feeds of ventilator waveforms and patient monitors as required.

\section{Case example 1: video calls with patients' loved ones}

Dr Louit Thakuria, ICU consultant: Supporting the family of a patient who is dying on the ICU is always extremely challenging. In normal circumstances, I considered it an essential part of my job to have these conversations in person whenever possible, explaining the gravity of the situation, encouraging families to spend time with their loved ones and offering whatever emotional support I could. This quickly became impossible as the pandemic grew, but the prospect of families having no contact with their critically ill relatives was frankly unacceptable. Our bedside webcams, however, offered an alternative approach.

The daughter of one man, who was rapidly developing refractory multiorgan failure, found phone conversations especially challenging. In this case, physical visiting was impossible, so we subsequently arranged a video call with the patient's relatives. Our clinical psychologist spent time preparing the family as best she could, and we then turned on the bedside webcam. We talked 
through the situation and explained what we were trying to do. We were able to show the family that we were truly doing all we could to save their loved one's life. The family were also able to see that he was comfortable, not in distress or pain, and was not alone. It allowed his wife and daughter to speak to him, to weep, and to say goodbye. A few days later, I received a particularly touching letter from that family thanking us for all that we had done.

Helen McGuire, ICU nurse: We soon recognised that all patients' families could benefit from the availability of video calls, so long as they were prepared in advance and staff understood the system. Some of the phone calls with awake patients involved large, multigenerational families, each member of which was able to have a short interaction with their loved one (or even sing to them), bringing both parties a sense of connection during this enforced separation. For one family, this helped them to truly understand that their father was critically unwell in our hospital, but also enabled them to provide much needed reassurance when he did wake with delirium and reduce the need for further sedation.

We would never choose to support families in patients with video calls rather than in-person visits. They do not allow for the norms of hands-on support to families in extremely emotional circumstances; a reassuring hug or the offer of tissues. But the pandemic did not present us with a choice. Our families were grateful simply to be able to speak to, and crucially see, their loved ones, and our staff felt reassured that we had done everything we could to support them.

\section{Case example 2: a specialty registrar's experience of using video technology}

Dr Rebecca Lewis, ICU specialty registrar: During the COVID-19 pandemic, I was working as a senior intensive care registrar at the Royal Brompton Hospital. This meant that, almost overnight, our ICU surged over capacity with very critically unwell, difficult to ventilate, intubated patients. Further changes also appeared, such as PPE, red zones and new areas of the hospital ventilating patients. At night, I was the senior doctor responsible for the management of these patients. However, the PPE and red zones became barriers to communication and made movement between zones challenging. This made it difficult to review deteriorating patients in multiple zones and provide support to less experienced members of the team.

The use of webcams, MS Teams and hand-held devices quickly allowed us to overcome these difficulties. As a registrar in the green zone doctor's office, I could be immediately contacted by any one of my juniors from within the red zone for advice and support. This was particularly useful in aiding the management of advanced ventilation during the pandemic. Using the technology, I was able to see all relevant real-time data for each patient including the vital signs and blood gases. In addition, the junior within the red zone could stand within the bed space on a video call to show me both the patient and the ventilator screen with flow loops. From this, I gained further clinical information to aid my decision making, while also being in continuous communication with both the bedside nurse and the junior doctor. I could easily communicate my decisions and changes to the management plan to the whole team involved and support the junior to optimise ventilation.

The technology allowed me to support juniors across many levels within the hospital as if I was at the bedside myself. This became a very powerful tool during the pandemic when, very quickly, the footprint of intensive care grew far beyond the walls of the original ICU with numerous critically unwell patients.

\section{Case example 3: nurse practice education using virtual ICU support}

Ian Naldrett, lead nurse for critical care education and training: One of the largest challenges in responding to the COVID-19 pandemic within our hospital setting is the re-deployment of staff to areas of work that are unfamiliar and not within their normal scope of practice. The key to assisting these staff was training. However, social distancing limited the options for faceto-face training and an alternative approach was required. As an education team, it was like running a marathon with your legs tied together; you need to facilitate learning so desperately but cannot use any of your traditional tools.

The rapid deployment of virtual ICU assistance presented an opportunity to provide this education online and interactively at the bedside through short sessions of teaching on relevant subjects that could be accessed safely, both remotely on a device from home or at the bedside. These sessions do not constitute 'e-learning.' Instead they are 'virtually facilitated' sessions in which the learner retains all the experience of being able to communicate and ask questions to experts to clarify any points.

We have retained the sessions beyond the peak of the pandemic and plan for them to remain as part of routine education for staff working in ICU. Indeed, they have evolved constantly to the changing needs of those accessing them. A simple innovation has proven to transform the reach of our education team in the most difficult circumstances. Not only did we feel that we had our marathon-running legs back, but we now had a jet pack to increase the accessibility and effectiveness of our teaching.

\section{Conclusions}

The COVID-19 pandemic presented many serious challenges and forced new ways of working in a rapidly changing clinical environment, facilitated by digital technology. The use of video software for multidisciplinary team meetings or outpatient consultations that are traditionally conducted face-to-face is well documented. ${ }^{6}$ It is now likely these changes to clinical practice are here to stay, beyond the lifecycle of the pandemic. However we have presented how new ways of working have extended into critical care, with three key areas of clinical practice transformed using readily available low-cost digital solutions during the COVID-19 pandemic:

$>$ communication and supervision within clinical teams

$>$ supporting the families of critically ill patients

$>$ clinical education.

The key strength of our system was its use of existing infrastructure, albeit for a purpose outside of its original design. This allowed us to incur negligible costs and implement it in a matter of days using pre-existing hardware and software with which staff were already familiar. In the vast majority of cases, staff were quick to recognise the benefits that the system offered, actively seeking implementation at the earliest opportunity in their clinical area. We found that the initial reticence of those less familiar with the technology was rapidly allayed once the system and accompanying processes were outlined. The fact that the team leading the project was multidisciplinary, incorporating users 
of the system in the ICU, certainly further helped to encourage widescale adoption.

Much of our technologies are also widely utilised across the NHS and we therefore believe our system could be adopted relatively easily and affordably in other clinical settings. There are, however, important considerations. Maintaining security of confidential patient data is of paramount concern. To this end, robust clinical and information governance guidelines are required to ensure compliance with GDPR and to protect patient confidentiality in accordance with the same Caldicott principles that apply to any other form of clinical communication. ${ }^{7}$ The Information Commissioner's Office have also provided advice supporting the use of video conferencing software to help patients to communicate with their families, with similar comments from NHSX.,9

We recognise that the system is not perfect. The use of video communication remains new in the critical care clinical environment and does require some adjustment by staff. Transmitting waveforms by webcam does not provide the same degree of resolution as a comprehensive, integrated 'e-ICU.' Video calls with family members can be particularly challenging when patients are critically ill and may not survive, and we wholly recognise that such calls can never fully replace physical contact. There have, inevitably, been occasions when the connection on a call has been temporarily lost, or several attempts to achieve a successful call have been required, adding to an already acutely stressful set of circumstances. Such situations are also difficult even for experienced staff and supporting their wellbeing after emotional calls must be a high priority.

Yet the overarching benefits are clear. Embracing digital solutions in the clinical workplace presents us with a unique opportunity to connect patients, families and clinicians; and these are connections that could benefit patient care beyond the challenges of the COVID-19 pandemic. The need to provide high-quality interactive clinical education will not disappear with the COVID-19 pandemic, and we fully intend to retain our virtually facilitated training sessions on ICU. It is easy to envision a future beyond COVID-19 where digital communication will complement all types of traditional clinical interactions, allowing us to create a more connected health service for clinicians and patients.

\section{Acknowledgements}

We were fortunate to have multiple donations in response to our appeals for webcams from members of the general public, The Royal
Brompton and Harefield Charity, Techbuyer Europe, The University of Reading, Expleo and the Grosvenor Estate.

\section{References}

1 Yoo BK, Kim M, Sasaki T, Hoch JS, Marcin JP. Selected use of telemedicine in intensive care units based on severity of illness improves cost-effectiveness. Telemed ] E Health 2018;1:21-36.

2 Schwamm LH, Erskine A, Licurse A. A digital embrace to blunt the curve of COVID19 pandemic. NPJ Digital Medicine 2020;3:64.

3 Vilendrer S, Patel B, Chadwick W et al. Rapid deployment of inpatient telemedicine in response to COVID-19 across three health systems. J Am Med Inform Assoc 2020;7:1102-9.

4 Barbash B], Sackrowitz RE, Gajic O et al. Rapidly Deploying Critical Care Telemedicine Across States and Health Systems During the Covid-19 Pandemic. NEJM Catalyst Innovations in Care Delivery 2020.

5 Intensive Care Society. ICS guidance on the use of video communication for patients and relatives in ICU. ICS. www.ics. ac.uk/ICS/Pdfs/COVID-19/Guidance_on_the_use_of_video_ communication_for_patients_and_relatives_in \% 20_ICU.aspx [Accessed 01 August 2020].

6 British Medical Association. COVID-19: video consultations and homeworking. BMA, 2020. www.bma.org.uk/advice-and-support/ covid-19/adapting-to-covid/covid-19-video-consultations-andhomeworking [Accessed 01 August 2020].

7 The UK Caldicott Guardian Council. The Caldicott principles. The UK Caldicott Guardian Council. www.ukcgc.uk/manual/principles [Accessed 01 August 2020].

8 Information Commissioner's Office. Health, social care organisations and coronavirus - what you need to know. ICO. https://ico. org.uk/global/data-protection-and-coronavirus-information-hub/ data-protection-and-coronavirus/health-social-care-organisationsand-coronavirus-what-you-need-to-know/\#health7 [Accessed 01 August 2020].

9 NHSX. FAQs for staff in health and care organisations. NHS, 2020. www.nhsx.nhs.uk/covid-19-response/data-and-informationgovernance/information-governance/faqs-staff-health-and-careorganisations [Accessed 01 August 2020].

Address for correspondence: Dr Louit Thakuria, Adult Intensive Care, Royal Brompton Hospital, Royal Brompton \& Harefield NHS Foundation Trust, Sydney Street, London SW3 6NP, UK. Email: louit@doctors.net.uk 